A CASE OF IDIOPATHIC ANAMIA; RECOVERY UNDER THE USE OF ARSENIC (FOWLER'S SOLUTION).

BY R. T. EDES, M. D.,

Professor of Materia Medica in Harrard Vniversity, and one of the Visiting Physicians at Boston City Hospital.

Since Dr. Addison first (and best) described a peculiar form of anæmia of unknown origin, calling it "idiopathic," but more especially since the disease received its German baptism at the hands of Biermer as "progressive pernicious," cases liave not been infrequently reported which with considerable regularity justified, in spite of all treatment, the later designation.

Since, however, cases have been observed where the diagnosis was open to no reasonable doubt, but which formed exceptions to this rule, it appears as if the earlier title, which expresses no opinion as to prognosis, were to be preferred.

The following case, though showing nothing new, does, I think, add one to the small number of those which have recovered, not from the use of either of the two methods of treatment which would at first sight suggest themselves, but which all experience in this disease has shown to be in most cases useless or worse,that is, first, large doses of iron, and, second, transfusion of blood, - but from a drug which, however difficult may be the physiological explanation of its action, has been empirically proved to possess great value in this as in other forms of anæmia, namely, arsenic.

J. A., aged forty-one, single, American, ship carpenter, entered the City Hospital on May 11, 1880. He is said to have had a "heavy cold" two years ago, and not to have been well since. Works at his trade at intervals. No further cause for his condition was discovered, although the records fail to show just how searching the cross-examination may have been, and it is not now possible to complete them.

He is a man of large frame, good muscular development, and though not inclined to obesity, like many subjects of this disease, yet by no means emaciated. His complexion is exceedingly pale, with hardly a trace of red, somewhat yellowish in hue, with the tint of anæmia but not of jaundice.

He complains of weakness, headache, dyspnoea on exertion, loss of appetite, and general malaise. His sight is dim, and he is somewhat deaf. His aspect is listless and apathetic. Has had a very slight cough without any expectoration. Epistaxis occasionally. Considerable pain in lumbar region. Not much vomiting, but food distresses him. Bowels usually costive.

There is but a slight amount of ascites now, but he says his bowels have been much larger.

No odema of feet or ankles at present, but they have been considerably swollen. He complains of palpitation.

Pulse is 90, the heart-sounds feeble, and there are systolic souffles both at the base and apex. The spiygmograph shows low tension. The spleen measures ten or eleven $\mathrm{cm}$. The lower edge can be felt by the fingers thrust deeply under the rib during inspiration. 'The hepatic dulluess exteuds a little below the border of the ribs.

May 12th. Urine slightly smoky, acid, 1024. Trace - of albumen. Sediment ; pus, blood, granular and very few epithelial casts. Mlay 14th. High colored, 1012. Albumen, slight trace, a few hyaline casts.

On May 12th he began to take tincture of chloride of iron, thirty minims every four hours. On May 17 th this was omitted, and Fowler's solution, five minims, substituted. Quinia was given for some time, citrate of magnesia and guarana for an occasional headache, and from time to time a cathartic.

At first his appetite was very small, and he had much nausea. A "liquid food" which had been sent to the hospital, which I think was largely composed of blood preserved by alcohol and aromatics, but which I found contained a large amount of albumen coagulable by heat, was used by the mouth, and, combined with pepsine, by the rectum.

May 22d. Afterwards eggs with pepsine were given as enemata. Soon after he made an unauthorized addition to his diet by eating a hearty dinner of ham and eggs.

On June 8th his urine contained nothing abnormal.

From this time on his appetite, strength, and complexion rapidly improved, until on July 4th his patriotism became so irrepressible that he left the hospital without leave, and I have been unable to get hold of him since. He is said by persons at his boarding place to have left for parts unknown, and to have been pretty well when he went away. Further tidings it has been impossible to obtain.

As detailed above the case presents but little to distinguish it from an ordinary convalescence, and a critic might suggest, in view of the first examination of urine and the history of some odema, that he had had a mild wephritis. In fact, he was for a short time supposed to be suffering from renal disease. This supposition, however, is negatived by the very rapid disappearance of all symptoms which could be interpreted in this way, even at a time when the patient was not in the least improving as regards the general condition, and the progress was regarded by himself and attendants as very unfavorable; and more especially by the state of the blood, which I did not report with the other symptoms, as the progressive improvement in its globular richness can be best seen by bringing the successive counts together.

The first examination of the blood showed in the most distinct manner to the naked eye its extreme poverty in red corpnseles. It appeared, as issuing from a puncture, of a pale pink color and extremely thin and watery.

Under the microscope the corpuscles were found not only very few in number, but deformed both as to size and shape. Many were much smaller and a few larger than the average. They were irregularly distorted, pear-shaped, globular, or nearly so, some with distinct tails as if a portion were being pinched off; others were distinctly and deeply cupped, and gare one the impression of the swelling of a distensible mass, tending to retain somewhat of its original shape, within an indistensible but flexible envelope. One would be likely to suppose that they might be deformed because of floating is a serum of density less than normal. In fact, I am inclined to think that a considerable part, if not all, of the deformity in shape was due to an altered relation between the density of the corpuscles and the serum in which they floated. This would very well account for one of the most frequent distortions observed, namely, a disk which had nearly become a sphere, but with two slight indentations, like a foot-ball nearly but not quite blown up. I have since noticed this same appearance to a less extent in the blood corpuscles from an anæmio patient probably with malignant disease, and a globular richness of a little over two million. A very good idea of the various forms assumed may be formed from the 
plates accompanying a very interesting jecture on this disease, by Dr. Finuy, in the British Medical Journal for January 3, 1880, which not only confirmed the diaguosis, but suggested the treatment apparently so successful.

The blood countings were made with a Gower's hæmocytometer, us arranged under Dr. Amory's direction.

It should be said that the difference between the first three countings is not beyond the usual limits of error, unless very great care is taken and a large number of fields counted; a somewhat tedious process in the midst of ordinary clinical work, and not altogether agreeable to eyes not of the strongest. The first two observations (1hree counts) probably signify that no material change had then taken place.

My instrument gives in presumably healthy blood five million or more red corpuscles to the cubic millimetre.

Red corpuscles in one cubic millimetre :-

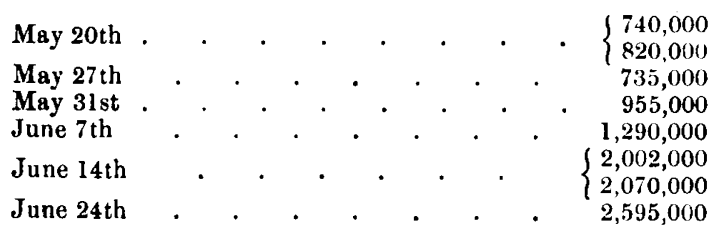

As the proportion of red corpuscles increased, that of the deformed ones diminished.

It has been quite generally acknowledged by all writers upon this disease that iron is very far from producing the favorable effect we might naturally anticipate, and in most cases utterly fails in usefulness.

One case, however, is reported by Dr. Byrom Bramwell, ${ }^{1}$ where the patient, who was unable to bear arsevic, recovered under the use of iron. The latter drug, however, has been the most essential part of the treatment in those other few cases, reported by Dr. Bramwell and by Dr. Finny, ${ }^{2}$ which have recovered, and it is difficult to avoid assigning to it an important share in the exceptional result.

In this case the dose was not large, being five minims of Fowler's solution at first, afterwards increased to seven and one half; suspended for a day or two on account of swelling beneath the eyes, and resumed at a little smaller dose.

Transfusion of blood has been a resource to which the thoughts of the physician, in presence of so hopeless a condition, would naturally turn, but it has proved a delusive one. It has never been of any permanent value, and Dr. Pepper has shown" that it is "not free from grave danger."

\section{RECENT PROGRESS IN DERMATOLOGY.4}

By E. WiggLesworth, M. D.

NO GENERAL TREATMENT FOR SYPHILIS BEFORE THE GENERAL SYMPTOMS.

Professor v. Sigmund called attention ${ }^{5}$ last year to the vast experience upon which he based his dictum, repeated in his article of this year, ${ }^{6}$ upon syphilis,

1 Edinburgh Medical Journal, November, 1877.

2 Loc. cit.

8 American Journal Medical Science, October 1875.

4 Concluded from page 349 .

5 Wien. med. Wochensebr., No. 10, 1879.

6 Wien. med. Wuchenschr., No. 16, 1880, et seq. namely, no general symptoms, no general treatment. Mercury, potassic iodide, etc., he considers useless and often harmful, if given for treatment of the initial sclerosis before subsequent constitutional manifestations are present. The initial lesion is not healed thereby, the consequences of the infection are not in any way arerted, the general health may be impaired. There is no "humanity" in too early treatment. Unity or duality of the virus is by no means a settled question, and there is very often serious difficulty or impossibility in deciding between the simple venereal ulcer and the initial sclerosis of syphilis. The latter, like the former, may disappear without treatment; its consequences always follow. And yet forty per cent. of healthy people attacked by syphilis suffer so little subjectively or oljectively that neither they nor their physicians necessarily become aware of the existence of the virus in their systems. Too early general specific treatment is a paudering of medical science to lay superstition. By local and general hygienic treatment, however, much is to be gained. The existence of other general or of local venereal diseases must be particularly regarded. Diet is to be considered, and especially is the importance of absolute cleanliness of every part of the person to be borne in mind. Here individual portions of the body liable to be affected later by lesions belonging to the secondary stage, and also individual idiosyncrasies, must not be forgotten. It is rarely sufficient to lay down general rules, in the foolish hope that the average patient may possess by instinct the knowledge the physician has been years in acquiring; minute details are to be taught and to be enforced by frequent supervision. This supervision is needful on account of the recklessness, the bad habits already formed, the stupidity, and the helplessness of the patient, and also from the fact that, owing to the privacy insisted upon, many means of affording relief are excluded, and it is indeed a test of patience, and usually a thankless labor. These facts show the need of special hospitals for the treatmeut of syphilitic patients. The parts to be daily inspected and most carefully cleaned are those liable subsequently to erosions, ulcerations, pustules, etc., such as the genitals, anus, vulva and vagiua, scalp, mouth, nares, throat, umbilicus, nipples, axillæ, elbows, and kuees, and the belly, breast, and back, where the erythema is most liable to first present itself. The neglect of cleauliness of the entrances to the body is often followed by quite serious consequences, to the mouth and throat in particular. Do not allow violent changes from old habits. Gauge the diet by the exertion demanded by daily life. Business must not be allowed to exhaust the patient. Good vertilation is needed, but no special raising of the temperature of the house. Scrofulosis, tuberculosis, and auæmia in youth, in age, and in preguant womeu call for special attention.

COMPLETE AND UNIVERSAL ALOPECIA FOLLOWING FRIGHT.

Total baldness coming on rapidly is usually the rèsult of severe fevers, and is followed by entire restoration to the normal condition. A case of Frédet ${ }^{7}$ is cited, ${ }^{8}$ however, which may perhaps be regarded us a unique one. A healthy Italian blonde, aged seventeen, lymphatic, with exceptionally profuse hair, was sewing at her window. Suddenly the Hoor fell in, leaving her

7 Annales de la Société de Méd de St. Etienne, Arch. gén., 1879. 8 Allg. med. Centr.-Zeitung, March 6, 1880. 